\title{
Correction: dehydratase mediated 1-propanol production in metabolically engineered Escherichia coli
}

\author{
Rachit Jain and Yajun Yan ${ }^{*}$
}

\section{Correction}

After publication of this work [1], we have noticed accidental errors that were introduced during our revision process. In our revision process, to address review comments we reduced the number of significant digits for the results of our enzyme assay experiments. As we reduced the number of significant digits to two in Tables 1 and 2, we overlooked the corresponding values mentioned in the text of "Results and Discussion" section discussing the "Methylglyoxal Synthase Assay" and "Secondary Alcohol Dehydrogenase Assay" [1]. In order to maintain consistency between the text and the tables, we would like to correct Tables 1 and 2 by increasing the number of significant digits up to four, which were used for our original submission. These changes will in no manner affect the outcome/interpretation of the experiments as described in the original publication and will not affect the merit of this work. In addition, we would like to
Table 2 Specific activity and $K_{m}$ determination of the secondary alcohol dehydrogenases

\begin{tabular}{lcccc}
\hline Gene & \multicolumn{2}{c}{ Methylglyoxal } & \multicolumn{2}{c}{ Hydroxyacetone } \\
\cline { 2 - 5 } & $\begin{array}{c}\text { Specific Activity } \\
(\mathbf{U} / \mathbf{m g})\end{array}$ & $\begin{array}{c}\boldsymbol{K}_{\boldsymbol{m}} \\
(\mathbf{m M})\end{array}$ & $\begin{array}{c}\text { Specific Activity } \\
(\mathbf{U} / \mathbf{m g})\end{array}$ & $\begin{array}{c}\boldsymbol{K}_{\boldsymbol{m}} \\
(\mathbf{m M})\end{array}$ \\
\hline gldA & $2.456 \pm 0.001$ & $68.24 \pm 0.05$ & $0.912 \pm 0.008$ & $10.47 \pm 0.55$ \\
\hline budC & $3.718 \pm 0.066$ & $0.78 \pm 0.03$ & $4.970 \pm 0.007$ & $1.83 \pm 0.63$ \\
\hline
\end{tabular}

The decrease in absorbance of $\mathrm{NADH}$ at $340 \mathrm{~nm}$ was recorded and used for calculations using the substrates methylglyoxal and hydroxyacetone. Substrate concentration was varied from $20 \mathrm{mM}-120 \mathrm{mM}$. 1 unit (U) was defined as the amount ( $\mu$ moles) of product formed per unit time (min).

modify the "Competing Interests" as below. The authors apologize for any inconvenience caused thereof.

\section{Authors' contributions}

YY and RJ conceived the study. RJ performed the experiments under the guidance of YY. An equal contribution by YY and RJ was made for literature review and drafting of the manuscript. Both authors read and approved the final manuscript.

Table 1 Methylglyoxal synthase assay results

\begin{tabular}{|c|c|c|c|}
\hline$m g s A$ source & Specific Activity (U/mg) & $K_{m}(\mathrm{mM})$ & Specific Activity $/ K_{m}(\mathrm{U} / \mathrm{mg} / \mathrm{mM})$ \\
\hline C. acetobutylicum & $0.0541 \pm 0.0042$ & $0.776 \pm 0.005$ & 0.0697 \\
\hline B. subtilis & $0.0561 \pm 0.0031$ & $0.473 \pm 0.070$ & 0.1186 \\
\hline C. difficile & $0.0597 \pm 0.0039$ & $1.439 \pm 0.060$ & 0.0415 \\
\hline E. coli & $0.1242 \pm 0.0069$ & $1.418 \pm 0.120$ & 0.0876 \\
\hline T. thermophilus & $0.0161 \pm 0.0004$ & $2.118 \pm 0.070$ & 0.0076 \\
\hline K. pneumoniae & $0.0165 \pm 0.0009$ & $2.820 \pm 0.300$ & 0.0058 \\
\hline P. fluorescens & $0.0133 \pm 0.0082$ & $1.560 \pm 0.020$ & 0.0085 \\
\hline R. eutropha & $0.0052 \pm 0.0004$ & $0.700 \pm 0.030$ & 0.0074 \\
\hline
\end{tabular}

Substrate dihydroxyacetone phosphate concentration was varied from $0.15 \mathrm{mM}$ to $1.5 \mathrm{mM}$ for all reactions. 1 unit (U) was defined as the amount ( $\mu$ moles) of methylglyoxal formed per unit time (min).

\footnotetext{
*Correspondence: yajunyan@uga.edu

Biochemical Engineering Program, Faculty of Engineering, 601B Driftmier Engineering Center, University of Georgia, Athens, GA 30602, USA
}

\section{Competing interests}

The University of Georgia has filed a United States provisional patent on this technology. 
Received: 16 March 2012 Accepted: 30 March 2012

Published: 30 March 2012

\section{Reference}

1. Jain $R$, Yan Y: Dehydratase mediated 1-propanol production in metabolically engineered Escherichia coli. Microbial Cell Factories 2011, 10:97.

doi:10.1186/1475-2859-11-38

Cite this article as: Jain and Yan: Correction: dehydratase mediated 1propanol production in metabolically engineered Escherichia coli. Microbial Cell Factories 2012 11:38.

Submit your next manuscript to BioMed Central and take full advantage of:

- Convenient online submission

- Thorough peer review

- No space constraints or color figure charges

- Immediate publication on acceptance

- Inclusion in PubMed, CAS, Scopus and Google Scholar

- Research which is freely available for redistribution

Submit your manuscript at www.biomedcentral.com/submit
() Biomed Central 